\title{
POLY[(VINYL ALCOHOL) - (STEARIC ACID)] SYNTHESIS AND USE IN LAVENDER OIL CAPSULATION
}

\author{
Arif Ozcan (D), Emine Arman Kandirmaz \\ Marmara University, School of Applied Sciences, Printing Technologies, Istanbul, Turkey
}

\begin{abstract}
Block polymers are used frequently in medicine, nanotechnology, paint, cosmetic and many other fields. Generally, one of the blocks produced is hydrophilic and the other hydrophobic. With amphiphilic polymers, molecules in the lipophilic structure can be encapsulated. Encapsulation is being used industrially for the reason that it is easier to transport a substance chemically without deterioration and is less affected by environmental effects. Amphiphilic block copolymers composed of hydrophilic and hydrophobic monomer units are used in micellization. Copolymers can form different morphological structures, which can be repeated under controlled conditions, depending on the composition of the block copolymer in the aqueous medium, the concentration of the copolymer in the medium, the interactions between the hydrophilic chains forming the shell, the addition of the acid, base or salt, the organic solvent used, the polarity of the solvent used and the relative solubilities of the blocks in the solvent. In these systems, while the core acts as a repository that allows the active substances to be dissolved, the shell part provides the hydrophilic property to the whole system. With amphiphilic polymers, molecules in the lipophilic structure can be encapsulated. In the first part of this work, stearic acid substituted polyvinyl alcohol-hydrophilic lipophilic polymer was synthesized with acidic esterification reaction and the chemical structure of the polymer enlightened with ATR-FTIR. ${ }^{1} \mathrm{H}-N M R$ method was used to determine the composition ratio of the polymer. In the second part of the study, lavender oil was added to the obtained polymer system and encapsulation was carried out after the interaction of the lavender oil and lipophilic end of polymer. The obtained capsule size analysis was performed by SEM. At the end of the work, paper coating formulations were prepared with microcapsules containing lavender oil and coated on standard office paper. The color and gloss properties of the coatings are measured. The results showed that the stearic acid substitute PVA polymer could be used in lavender oil encapsulation and made a suitable encapsulation for paper coatings.
\end{abstract}

Key words: block polymer, encapsulation, lavender oil, stearic acid, PVA

\section{INTRODUCTION}

Microcapsulation is the process in which the solid, liquid or gaseous compounds are taken up with a film material around them (Mars et al, 1990; Dubey et al, 2009) where the inner material is called the core, the corona, the active substance, and the outer material is called the shell, shield or wall. The capsule used is inert to the internal structure. so that the core structure is protected without degradation (Thies, 1996). The entire microencapsulation process essentially involves three separate processes that are separate from each other. The first process is to create a wall layer around the interior material. Second, the inner material is prevented from escaping from the formed outer wall layer. Besides, the outer wall layer should also prevent the ingress of unwanted materials which may damage the material inside. It takes place during the third process when the inside material is taken out at the beginning and at the right time This technique has been employed in a diverse range of fields from chemicals and pharmaceuticals to cosmetics and printing (Xiang et al, 1998; Cummings et al, 1996; Preston et al, 2001).

Microcapsules having a size of 1-100 $\mu \mathrm{m}$ are formed by coating an active inner material with a membrane which does not react with this substance (Moutinho et al, 2009). Microencapsulation of odor, drugs, vitamins, oils etc. protects the active material from oxygen, light, moisture, heat factors. It facilitates the transport of the active substance and also prevents it from evaporating during the stock and market. At the same time, it provides the spreading of the active substance in desired amount by the help of controlled release mechanisms. Many methods can be used to release active substance. Some of those; $\mathrm{pH}$, electric current, ultrasound, heat etc.

The commonly used microcapsulation techniques are coacervation, (Cost E32, 2002; Pruszynski, 2003; Moutinho, 2009) interfacial polymerization (Moutinho et al, 2011; Zhu et al, 2012; Suave et al, 2006) and "in situ" polymerization (Singh et al, 2010; Mervosh et al, 2009). Release mechanism, formation conditions, particle size, stability, usage area etc. effects microcapsule production technique. Microencapsulation technology has a lot of applications such as including pharmaceutical, chemical, food, cosmetics, perfumery, textiles, agriculture, etc. (Jegat et al, 2000; Soper et al, 2000). 
Lavender oil is an odour that it is used in cosmetic, pharmacy, antimicrobial usage, and aromatherapy (Ascheri et al, 2003) lavender oil have been used perfumery industry as a pleasant fragrance or as an antimicrobial agent. But lavender oil is high volatile and gives reaction with light, oxygen and heat. The microcapsulation is use to prevent this effects (Mizuno et al, 2005; Ouall et al, 2006).

The paper industry has to increase paper quality due to the increased customer demands and competition (Kandirmaz et al, 2018). The final quality requirements of the paper in the hands of the end user depend on the surface properties, and in recent years the paper industry has focused on improving the paper surface properties and answer the customer demands (Ozcan, 2016; Aydemir et al, 2013). In addition to improving the printability of paper, the paper is attracting and attracting features. Some of the charm attributes are colour changing paper, odorous paper, effect paper. The easiest way to impart a paper smell is to add a scented compound to the paper production process. However, most perfumes undergo oxidation, losing smell easily in an open environment and having a yellowish colour. In addition, oil compounds, which can be used for permanent odour, are not readily dispersed in an environment containing inorganic materials such as paper and adversely affect the production process. For this reason, the odour can be microcapsulated to facilitate the use of scents in paper production so that the surrounding perfume can not evaporate, the paper can not change the colour and the negative properties are reduced (Hirech et al, 2003; Jang et al, 2005; Brown et al, 2003; Chograni et al, 2010; Madene et al, 2006; Xiang et al, 1998; Pruszynski, 2003). The use of microcapsules in paper production can be accomplished by two methods, such as the addition of during the production and the coating on the paper surface. It is easier to apply with the coating on the paper surface because the homogenization process takes off and the production process is simplified.

For this purpose, stearic acid substituted PVA polymer was synthesized in this study and lavender microencapsulation with this polymer was performed. The coating formulations prepared with this microcapsule and cationic starch, papers coated with this formulation. The surface and printability properties of the resulting coatings were investigated.

\section{METHODS}

PVA, Stearic Acid, Citric acid, Ethanol, Sulphuric acid were obtained to Sigma Aldrich. Firstly, the wall material was synthesized. PVA (40 mmol), $200 \mathrm{~mL}$ of distillate water and stearic acid (10 mmol) were loaded to a three-necked glass flask equipped with a nitrogen inlet, a magnetic stirrer, a reflux condenser, and a thermometer. With the help of a dropping funnel, $1 \mathrm{ml} \mathrm{H}_{2} \mathrm{SO}_{4}$ was added drop by drop with $250 \mathrm{rpm}$ stirring at $80{ }^{\circ} \mathrm{C}$ in an oil bath. The mixture was refluxed at $80^{\circ} \mathrm{C}$ for 24 hours. The solution was participated with ethanol. Substituted polymer was filtered and dried overnight at room temperature in a vacuum incubator. In the capsulation study; $1 \mathrm{~g}$ stearic acid substituted PVA polymer was dissolved in $50 \mathrm{ml}$ distillate water, 2 hour stirred and the $1 \mathrm{ml}$ lavender oil was added onto the solution. After 1 hour $4 \mathrm{~mL} 1 \mathrm{M}$ citric acid was added the mixture and stirred 2 hours $500 \mathrm{rpm}$. Obtained capsules are filtered and dried. The obtained capsules chemical structure was enlightened with ATR-FTIR the morphological structures determined with SEM.

Cationic starch based surface coatings were applied to paper. The parameters of base paper used in this study are given in Table 1.

Table 1: Parameters of the base paper used in the study

\begin{tabular}{lll} 
Properties & Standard & Paper \\
\hline Grammage $\left(\mathrm{g} / \mathrm{m}^{2}\right)$ & ISO 536 & 80 \\
Thickness $(\mu \mathrm{m})$ & TAPPI T411 & 190 \\
Whiteness $(\mathrm{D} 65 / 10)(\%)$ & ASTM E313 & 99 \\
Gloss (TAPPI 60\% $\left./ 75^{\circ}\right)$ & T480 om-92 & 4.9 \\
Yellowness & ASTM E313 & 0.06 \\
\hline
\end{tabular}

The sizing formulation applied consisted of $7.5 \%$ concentration of cationic starch, which was heated up to $90{ }^{\circ} \mathrm{C}$, and the resulting hot-surface coating solution was cooled to $60{ }^{\circ} \mathrm{C}$ and then applied on to the paper surface using the MEYER rod with rod number 2 in a laboratory-type paper coating machine. The same 
preparation process was applied to the microcapsule added sizing formulation, which was the other section of this study, but 2.5\% Microcapsule was also added to the first mixture. The formulation was applied to the paper surface using with a laboratory-type paper coating machine under laboratory conditions.

CIE L*a*b* values of The original, sized and microcapsule sized papers were measured using X-Rite eXact hand-held spectrophotometer according to ISO 12647-2:2013, between a spectral range of $400 \mathrm{~nm}$ to 700 $\mathrm{nm}$, under a D50 light source, $2^{\circ}$ observer, polarized filter, and 0/45-degree geometry. The colour differences formula was given below. Calculations were made by taking the average of five measurements. $\Delta \mathrm{L}^{*}, \Delta \mathrm{a}^{*}, \Delta \mathrm{b}^{*}$ : Difference in $\mathrm{L}^{*}, \mathrm{a}^{*}$, and $\mathrm{b}^{*}$ values between specimen colour and target colour. Lightness is represented by the $L^{*}$ axis which ranges from White to Black. The red area is connected to the green by the $a^{*}$ axis, while the $b^{*}$ axis runs from yellow to blue.

$\Delta E=\sqrt{\Delta L^{2}+\Delta a^{2}+\Delta b^{2}}$

Gloss measurements were made with BYK-Gardner GmbH glossmeter according to ISO 2813:2014 60 geometry.

\section{RESULTS AND DISCUSSION}

Stearic acid substitute polyvinyl alcohol was prepared via acidic esterification (fisher esterification) technique according to Figure 1.

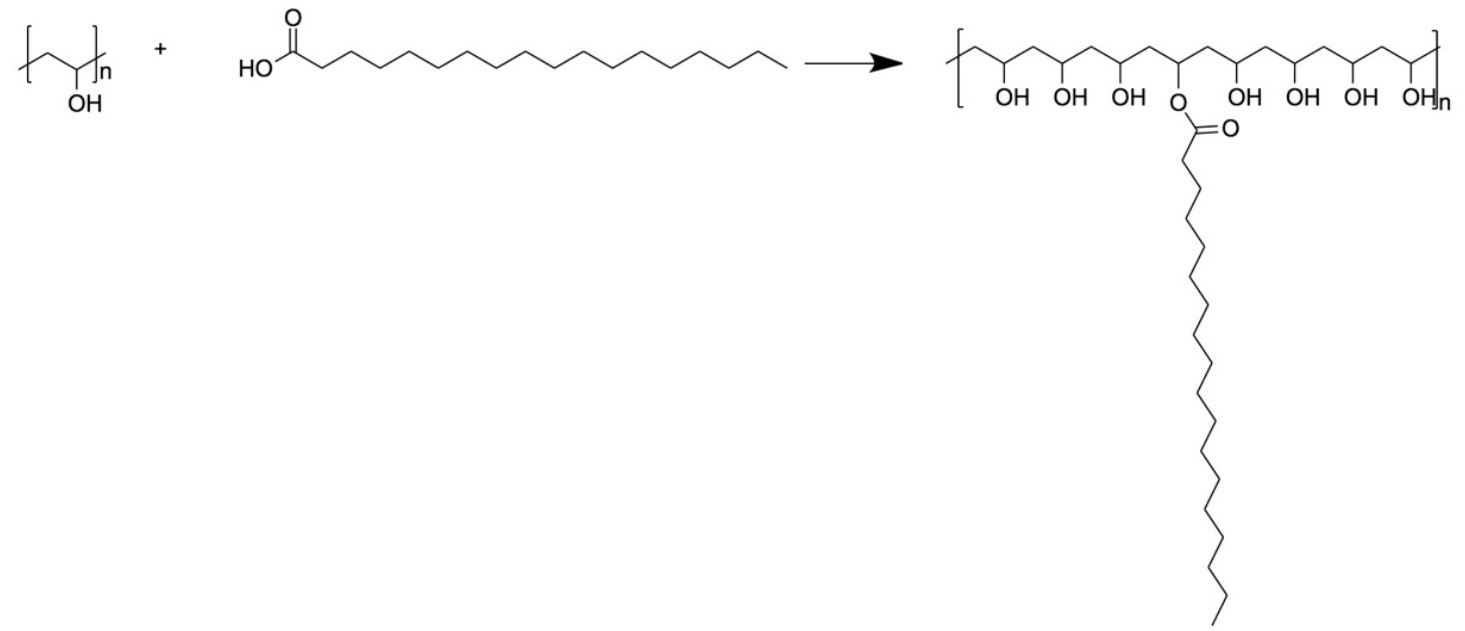

Figure 1: The synthesis of stearic acid substitute polyvinyl alcohol

ATR-FTIR and ${ }^{1} \mathrm{H}-\mathrm{NMR}$ spectra was confirmed the expected structures. In Figure 2, the characteristic ${ }^{1} \mathrm{H}-$ NMR signals of stearic acid substitute polyvinyl alcohol can be seen. The proton on the stearic acid bounded carbon proton signal for Stearic acid substitute polyvinyl alcohol structure was appeared at 4,69 ppm in Figure 2. Additionally, the spectrum also showed characteristic peaks for proton of the hydroxyl-bonded carbon at $3.59 \mathrm{ppm}$, Symmetric protons on to PVA at $1.72 \mathrm{ppm}$, Symmetric protons on stearic acid at 1,23 ppm, Symmetric protons on stearic acid first carbon at 2,25 ppm and at 1.67-0,84 ppm, the protons at the end respectively. It is clear that, the synthesis was successfully realized. When the area ratios of the peaks at $4.69 \mathrm{ppm}$ and $3.59 \mathrm{ppm}$ are examined, it is calculated to have 1: 8 ratios. Accordingly, the amount of substitution was determined to be $11 \%$. 


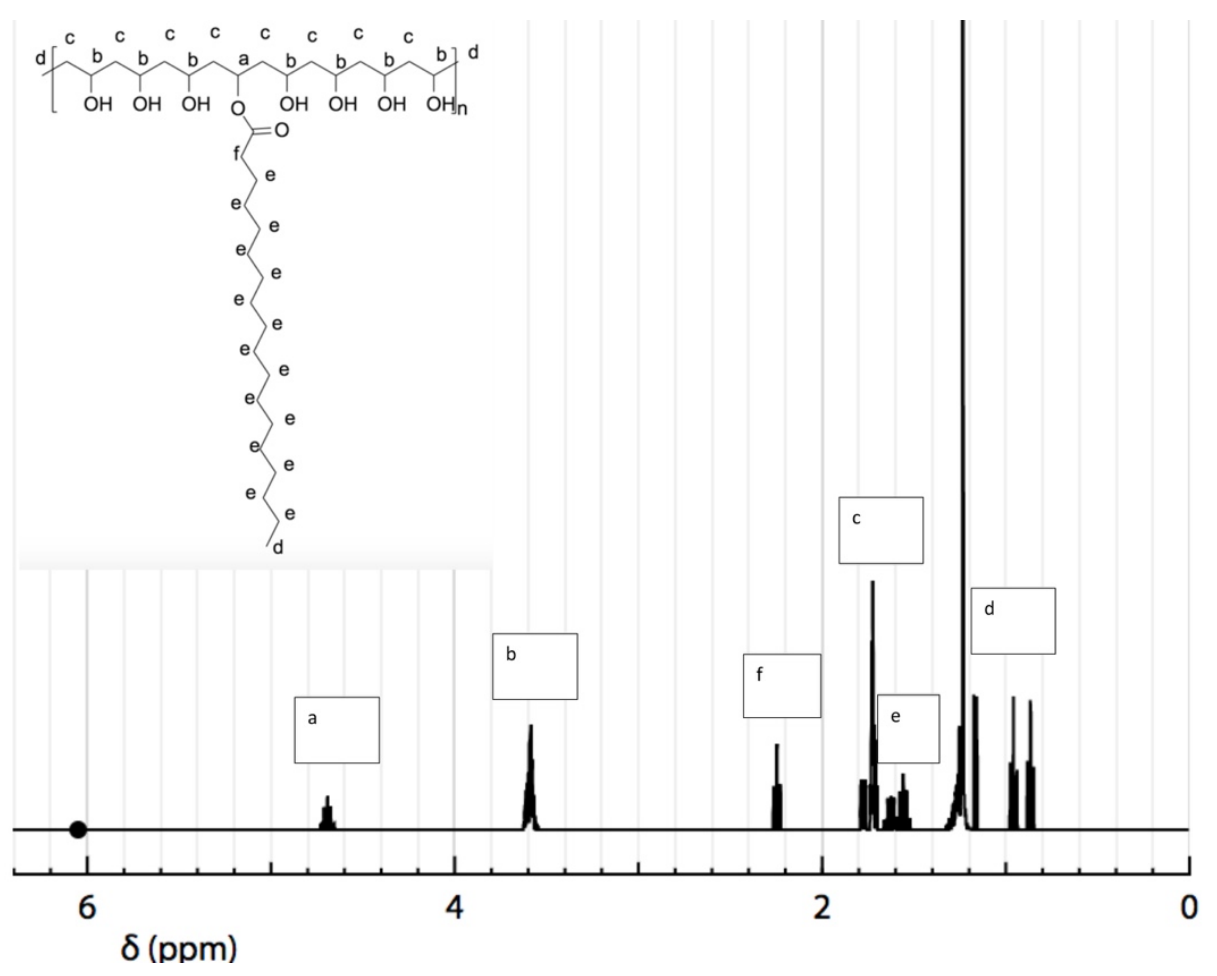

Figure 2: ${ }^{1} \mathrm{H}-\mathrm{NMR}$ spectra of stearic acid substitute polyvinyl alcohol

Moreover, ATR-FTIR analysis supports the ${ }^{1} \mathrm{H}-\mathrm{NMR}$ results. Figure 3 a showed that; the characteristic $\mathrm{C}-\mathrm{H}_{2}$ asymmetric alkyl stretching bond vibration band for PVA appeared at $2915 \mathrm{~cm}^{-1}$. Moreover, at $3307 \mathrm{~cm}^{-1}$ $\mathrm{OH}$ and Crystallization-sensitive band of PVA: $1090 \mathrm{~cm}^{-1}$. Figure $3 \mathrm{~b}$ showed that carbonyl group of stearic acid $1711 \mathrm{~cm}^{-1}$ and hydroxyl bands for free alcohol $3299 \mathrm{~cm}^{-1}$. Stearic acid substitute PVA was seen Figure 3c. C-O-C=O esteric bond vibration was appeared onto $1644 \mathrm{~cm}^{-1}$.

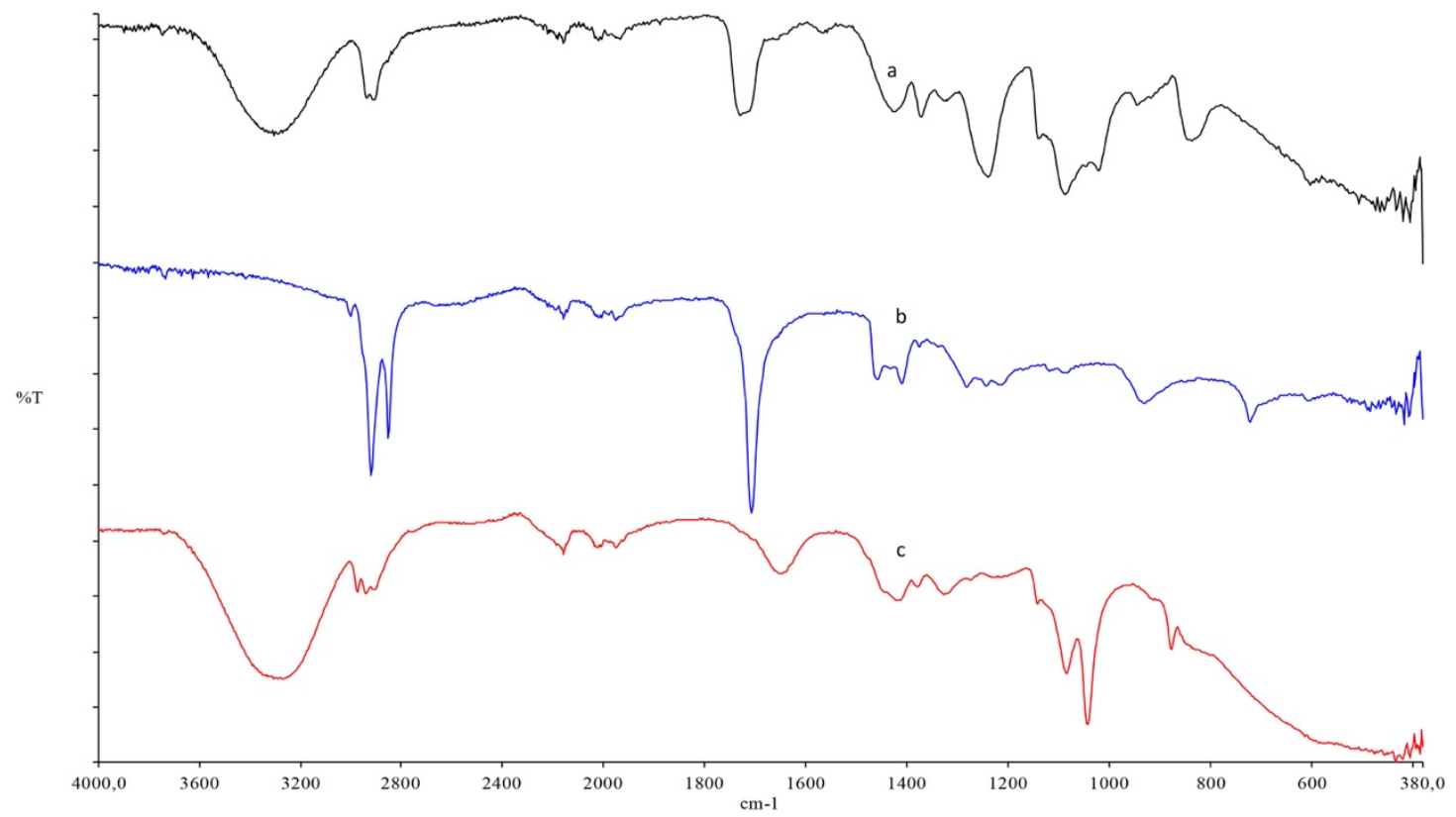

Figure 3: ATR-FTIR spectra of stearic acid substitute polyvinyl alcohol

Lavender oil capsulation with stearic acid substituted PVA polymer was carried out under acidic conditions. The chemical structure of the resulting microcapsules was illuminated by ATR-FTIR. When the ATR-FTIR 
spectra were examined, it was found that only the polymer in which the peaks of the oil were not seen, that is, the whole oil was encapsulated.

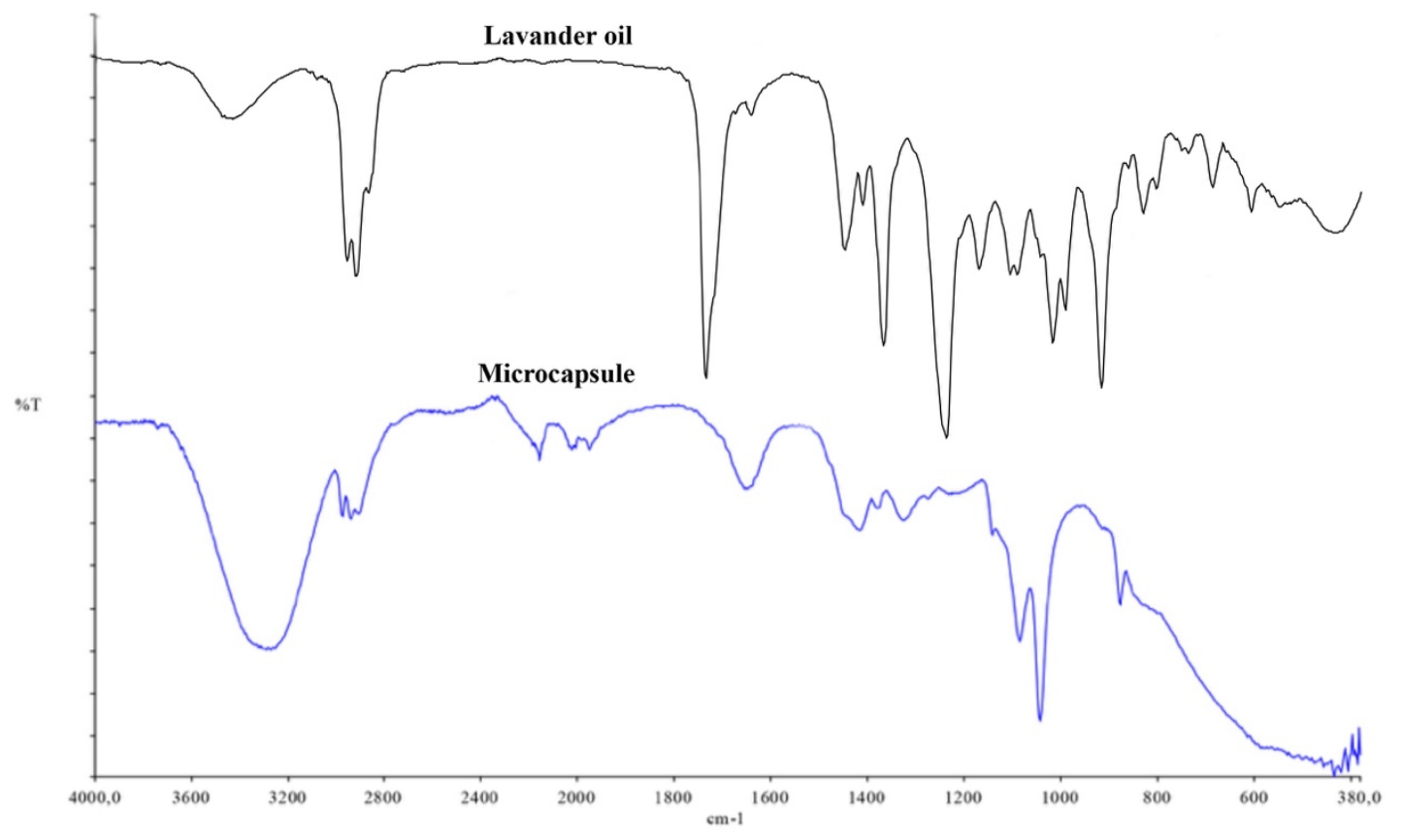

Figure 4: ATR-FTIR spectra of lavender oil microcapsule

When the microcapsule synthesis is examined, it is concluded that the encapsulation does not occur in basic $\mathrm{pH}$ only at extreme acidic $\mathrm{pH}$. In addition, when the SEM images were examined, it was determined that the microcapsule size was homogeneous and about $13 \mathrm{~nm}$.

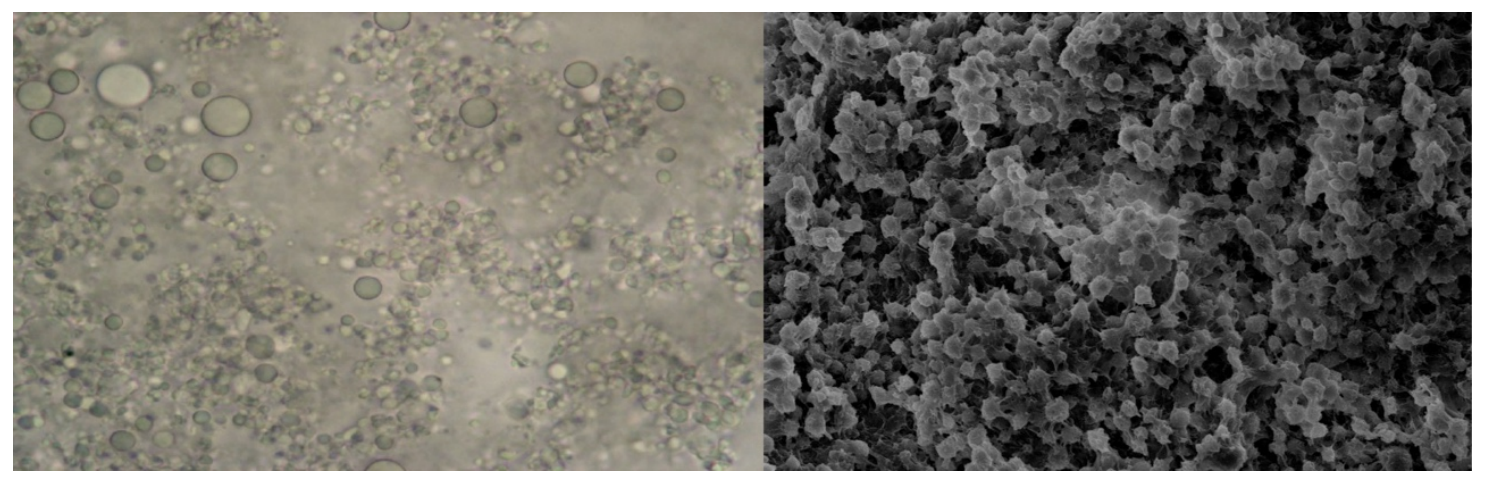

Figure 5: Optical and SEM images of lavender oil microcapsule

The formulations containing lavender oil capsules and cationic starch were coated on $80 \mathrm{~g} / \mathrm{m}^{2}$ paper. color and gloss values of coated and uncoated paper are given in the Table 2.

Table 2: Color and gloss values of paper and sized paper

\begin{tabular}{lccc} 
Properties & Uncoated paper & Sized paper & Microcapsule added sized paper \\
\hline L & 90,87 & 89,80 & 90,23 \\
a & 2,29 & 2,48 & 2,37 \\
b & $-8,02$ & $-8,3$ & $-8,14$ \\
Gloss (TAPPI 60 & 4,5 & 9,3 & 7,9 \\
\hline
\end{tabular}


When the table is examined, it is observed that the biggest change in the colour of the uncoated paper with the sizing process is colour goes to yellow. When the microcapsule was added to the starch, it was observed that the differentiation in the colour increased and the colour shifted to yellow more. When the colour differences between the obtained colours are calculated, it is concluded that the delta $\mathrm{E}$ value is in the values of 1,12 and 0,65 it is acceptable according to ISO 12647-2. When the gloss values were examined, it was found that the paper sized with starch had the highest gloss and microcapsule containing the second. All paper treated is higher than reference uncoated paper. This is due to the cationic starch applied to the environment. The reason for the decrease in the microcapsule is that the surface is slightly roughened by the capsules.

SEM images of uncoated paper, starch coated paper and microcapsule added starch coated paper are shown in Figure 6. When the Figure was examined, it was obtained that the microcapsule, where the surface was smoothed by applying starch, was spread uniformly on the surface and the coating was stable.

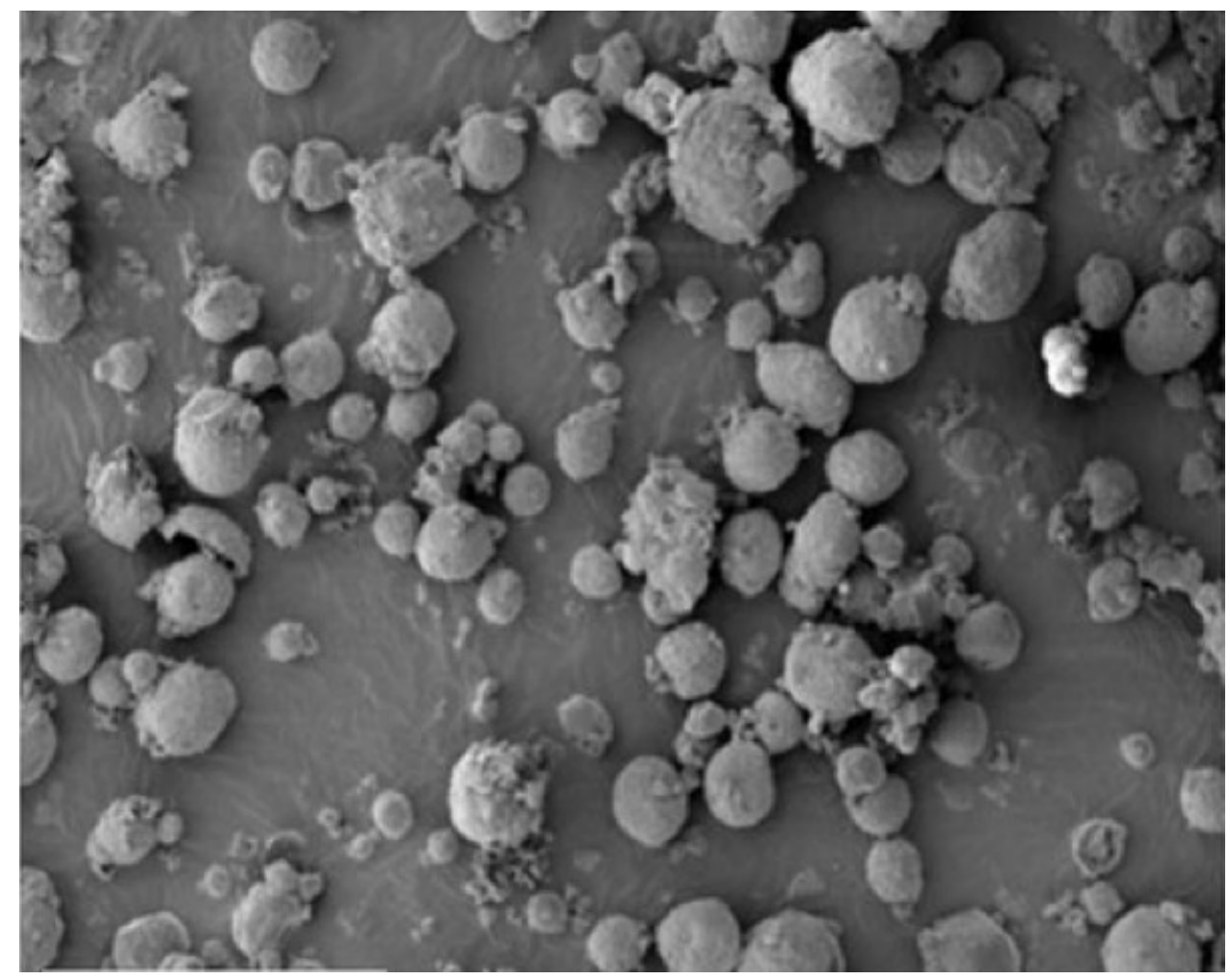

Figure 6: SEM images of microcapsule sized paper

\section{CONCLUSIONS}

Stearic acid substitute polyvinyl alcohol was synthesized by acidic esterification and it was used to prepare a new lavender microcapsule. ${ }^{1} \mathrm{H}-\mathrm{NMR}$ and ATR-FTIR was confirmed the expected structures. SEM images showed that the microcapsule sizes are mono dispersed. The sizing formulations are prepared with these microcapsules and apply onto paper successfully. Colour and optical properties of microcapsule sized papers are not better than uncoated paper but the difference is between the acceptable limits according to ISO 12647-2. Gloss is higher than uncoated paper because of extra cationic starch. And the surface of the microcapsule sized papers more uniform and not bursted.

\section{ACKNOWLEDGMENTS}

This work was supported by Research Fund of the Marmara University. Project Number: FEN-C-DRP$110718-0411$. 


\section{REFERENCES}

[1] Ascheri, D. P., Marquez, M. O., Martucci, E. T.: "Microencapsulação de óleo essencial de laranja: seleção de material de parede", Ciência e Tecnologia de Alimentos 23, 1-6, 2003. doi:10.1590/S0101-20612003000400002.

[2] Aydemir, C., Özomay, Z., Karademir, A., Kandırmaz, E. A.: "Effects of matte coating on the paper surface and print density", Science and Engineering of Composite Materials 20(2), 141-145, 2013. doi: 10.1515/secm-2012-0070.

[3] Brown, E. N., Kessler, M. R., Sottos, N. R., White, S. R.: "In situ poly (urea-formaldehyde) microencapsulation of dicyclopentadiene", Journal of microencapsulation 20(6), 719-730, 2003. doi: 10.1080/0265204031000154160.

[4] Chograni, H., Zaouali, Y., Rajeb, C., Boussaid, M.: "Essential oil variation among natural populations of Lavandula multifida L. (Lamiaceae)", Chemistry \& biodiversity 7(4), 933-942, 2010. doi: 10.1002/cbdv.200900201.

[5] COST E32: "Memorandum of understanding: characterization of paper surfaces for improved printing paper grades", (European Concerted Research Action COST Action E32, Brussels, 2002).

[6] Cummings, D. O., Lyons, A. V: "Influence of Engineered Kaolin on Web Offset", Proceedings of TAPPI Coating Conference 1996, (TAPPI Press, Nashville, Atlanta, 1996), page 16.

[7] Dubey, R., Shami, T.C., Bhasker Rao, K.U.: "Microencapsulation Technology and Applications", Defence Science Journal 59 (1), 82-95, 2009. doi: 10.14429/dsj.59.1489.

[8] Hirech, K., Payan, S., Carnelle, G., Brujes, L., Legrand, J.: "Microencapsulation of an insecticide by interfacial polymerisation", Powder Technology 130(1-3), 324-330, 2003. doi:10.1016/S0032-5910(02)00211-5.

[9] Jang, I. B., Sung, J. H., Choi, H. J.: "Synthesis of microcapsule containing oil phase via in-situ polymerization", Journal of materials science 40(4), 1031-1033, 2005. doi: 10.1007/s10853-005-6527-y.

[10] Jegat, C., Taverdet, J. L.: "Stirring speed influence study on the microencapsulation process and on the drug release from microcapsules", Polymer bulletin 44(3), 345-351, 2000. doi: $10.1007 / \mathrm{s} 002890050612$.

[11] Madene, A., Jacquot, M., Scher, J., Desobry, S.: "Flavour encapsulation and controlled release-a review", International journal of food science \& technology, 41(1), 1-21, 2006. doi: 10.1111/j.1365-2621.2005.00980.x.

[12] Mars, G. J., Scher, H. B.: "Controlled delivery of crop protecting agents", (Taylor and Francis, London, 1990.), page 65-90.

[13] Mervosh, T. L., Stoller, E. W., Simmons, F. W., Ellsworth, T. R., Sims, G. K.: "Effects of starch encapsulation on clomazone and atrazine movement in soil and clomazone volatilization", Weed Science 43(3), 445-453, 2009.

[14] Mizuno, K., Taguchi, Y., Tanaka, M.: "The effect of the surfactant adsorption layer on the growth rate of the polyurethane capsule shell", Journal of chemical engineering of Japan 38(1), 45-48, 2005. doi: $10.1252 /$ jcej.38.45.

[15] Moutinho, I. M. T., Kleen, A. M., Figueiredo, M. M. L., Ferreirea, P.J.T.: "Effect of surface sizing on the surface chemistry of paper containing eucalyptus pulp", Holzforschung 63(1), 282-289, 2009. doi: $10.1515 /$ HF.2009.046.

[16] Moutinho, I.M.T.: "Physical \& Chemical Interactions on Paper Surface - Impact on the Printability of Papers Produced with E. globulus Kraft Pulps", Coimbra University for the degree of Doctor of Philosophy in Chemical Engineering, 2009.

[17] Moutinho, I.M.T., Ferreira, P.J.T., Figueiredo, M. L.F.: "Paper surface chemistry as a tool to improve inkjet printing quality", Bioresources 6(4), 4259-4270, 2011.

[18] Ouall, L., Lahoussine, D.: WO, 2006027664A2, "Process for production nano-capsules containing a fragrance", 2006.

[19] Ozcan, A.: "Para-Amino Benzoik asit Eklenmiş Kaplamalarının Kağıdın Fiziksel ve Optik özelliklerine Etkisinin Incelenmesi", Proceedings of International printing technologies symposium 2016, (Printistanbul, Istanbul, Turkey, 2016), pages 331-340.

[20] Preston, J. S., Elton, N. J., Legrix, A., Nutbeem, C.: "The role of pore density in the setting of offset printed ink on coated paper," Proceedings of TAPPI. Advanced Coating Fundamentals Symposium 2001, (ACS, San Diego, USA, 2001), page 102. 
[21] Pruszynski, P.: "Recent developments in papermaking chemicals. In Chemical Technology of Wood", Proceedings of the International Conference Chemical Technology of Wood 2003, Pulp and Paper, (WPP, Bratislava, 2003), page 82-90.

[22] Pruszynski P.: "Recent developments in papermaking chemicals", WPP Keynote Lectures 82-90, 2003.

[23] Singh, M. N., Hemant, K. S. Y., Ram, M., Shivakumar, H. G.: "Microencapsulation: A promising technique for controlled drug delivery", Research in pharmaceutical sciences 5(2), 65-77, 2010.

[24] Soper, J. C., Yang, X., Josephson, D. B.: “U.S. Patent No. 6,106,875", Patent and Trademark Office, 2000.

[25] Suave, J., Dall'Agnol, E. C., Pezzin, A. P. T., Silva, D. A. K., Meier, M. M., Soldi, V.: "Microencapsulação: Inovação em diferentes áreas", Revista Saúde e Ambiente/Health and Environment Journal 7(2), 12-20, 2006.

[26] Thies, C. A: "Survey of microencapsulation processes. In Microencapsulation: Methods and industrial applications", (Marcel Dekker, New York, 1996), page 73-77.

[27] Ural, E., Kandirmaz, E. A.: "Potential of fish scales as a filling material in surface coating of cellulosic paper", Journal of applied biomaterials \& functional materials 16(1), 23-27, 2018. doi:10.5301/jabfm.5000378.

[28] Xiang, Y., Desjumaux, D., Bousfield, D., Forbes, M. F.: "Preprints of the microcapsule", The Preprints of the Pan Pacific and International Printing and Graphic Arts Conference 1998, (Quebec, Canada, 1998), page 85.

[29] Xiang, Y., Desjumaux, D., Bousfield, D., Forbes, M.F.: "The relationship between coating layer composition, ink setting rate and offset print gloss", Preprints of the Pan Pacific and International Printing and Graphic Arts Conference 1998, (Quebec, Canada, 1998), page 85.

[30] Zhu, G. Y., Xiao, Z. B., Zhou, R. J., Yi, F. P.: "Fragrance and flavor microencapsulation technology", In Advanced Materials Research 535, 440-445, 2012.

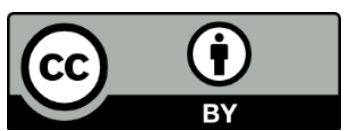

(C) 2018 Authors. Published by the University of Novi Sad, Faculty of Technical Sciences, Department of Graphic Engineering and Design. This article is an open access article distributed under the terms and conditions of the Creative Commons Attribution license 3.0 Serbia (http://creativecommons.org/licenses/by/3.0/rs/). 